\title{
Impact of plasma omentin levels on glucose metabolic disorders and early stage of endothelial dysfunction in elderly patients
}

DONG DONG YE

Shanghai Jiao Tong University School of Medicine https://orcid.org/0000-0002-7063-0329

\section{Wei Liang}

Shanghai Jiao Tong University Medical School Affiliated Ruijin Hospital

\section{Zhengjia Su}

Shanghai Jiao Tong University Medical School Affiliated Ruijin Hospital

Wenqi Pan ( $\square$ drpanwenqi@126.com )

Shanghai Jiao Tong University School of Medicine https://orcid.org/0000-0003-4517-6977

Original investigation

Keywords: Diabetes, Adipokine, Omentin, inflammatory, endothelial dysfunction

Posted Date: March 10th, 2020

DOI: https://doi.org/10.21203/rs.3.rs-16514/v1

License: (c) (i) This work is licensed under a Creative Commons Attribution 4.0 International License.

Read Full License 


\section{Abstract}

Background: Diabetes-induced endothelial dysfunction is a critical and initiating factor in the genesis of diabetic vascular complications. Omentin exerts direct influence on glucose metabolic disorders and vascular dysfunction. But the association between omentin and cardiovascular events reported by recent studies are paradoxical. Thus, we intend to investigate the relationship between omentin and vascular function in patients with different glucose metabolism states.

Methods: A total of 162 elderly patients were stratified into three groups by the status of glucose tolerance test [normal glucose tolerance (NGT), impaired glucose regulation (IGR), type 2 diabetes mellitus (T2DM)]. All participants underwent complete clinical work-up. Fasting blood samples were collected for biochemical measurement. Circulating omentin, adiponectin, interleukin-6 (IL-6) and tumor necrosis factor a (TNF-a) levels were detected by enzyme-linked immunosorbent assays. Endothelial and vascular smooth muscle function were evaluated by flow-mediated dilatation (FMD) and nitrate-mediated dilation test (NMD) respectively.

Results: Circulating omentin levels were lower in IGR group compared with NGT and were lowest in individuals with T2DM group ( $P=0.004)$. FMD has a significant and negative association with systolic blood pressure (SBP), glycosylated hemoglobin (HbA1c), fasting plasma glucose (FPG), fasting insulin and interleukin-6 (IL-6), while have a positive relationship with log (omentin) by bivariate correlation analysis in all subjects. After adjusting for traditional cardiovascular factors, log (omentin) was independent related factor that influenced FMD $(\beta=-0.346 \otimes P=0.01)$ in IGR subjects, but not in the subgroups NGT and T2DM.

Conclusions: A decreased omentin levels had been suggested to be a biomarker of glucose metabolic disorders and potentially cardiovascular risk. Higher circulating omentin level are independent determining factors for endothelial dysfunction in impaired glucose regulation (IGR) subjects suggesting and may be a compensatory mechanism for early diabetic vascular injury. which suggest omentin may act as potential markers for assessing endothelial dysfunction and identifying high risk of cardiovascular complications among asymptomatic prediabetic individuals.

\section{Background}

Type 2 diabetes mellitus (T2DM) represents a major risk factor for the development of cardiovascular diseases and ischemic stroke. A collaborative meta-analysis of 102 prospective studies indicated that diabetes confers about a two-fold excess risk for coronary heart disease, major stroke subtypes, and deaths attributed to other vascular causes, independently from other conventional risk factors. Besides, patients with diabetes are more likely to develop fatal myocardial infarction than those with normal blood glucose ${ }^{1}$. Although we have made several efforts in reducing cardiovascular morbidity and mortality with novel hypoglycemic drugs and regular management, convincing improvements in outcomes have not been achieved yet ${ }^{2}$.The morbidity and mortality rates of diabetes remain high. According to the latest 
data from the International Diabetes Federation (IDF), approximately $12 \%$ to $31.7 \%$ older people with type 1 and type 2 diabetes have coronary artery disease, which accounted for about $25 \%$ of vascular deaths in populations in developed countries ${ }^{3}$.

Endothelial and vascular smooth dysfunction are considered early abnormalities in the development of atherosclerotic process in cardiovascular diseases, with a significant prognostic role in high-risk population ${ }^{4}$. Impaired endothelial function has been consistently demonstrated in the macro-and microcirculation of individuals with type 2 diabetes, moreover, it appears long before symptoms. Unfortunately, a majority of older people with diabetes are unaware of having diabetes complications, thus, physicians always deal with diabetic patients with various complications rather than the early stage of the diseases. However, most complications can be detected in their early stages by screening programmes. Decreased bioavailability of nitric oxide is considered to be an important feature of vascular endothelial dysfunction, which can be quantified by the noninvasive technique of flow-mediated dilation (FMD) ${ }^{5}$. Nitrate-mediated dilation of brachial artery (NMD) using the exogenous nitrate administration to relax vascular smooth muscle (VSM), which is in an endothelium-independent manner, thus reflecting VSM function ${ }^{6}$. The noninvasive nature and repeatable characteristic of the technique allow its widespread usage in predicting cardiovascular outcomes in patients at high risk of developing cardiovascular diseases. However, there are also technical and interpretive limitations of this technique, which limit its widely clinical application.

Normalization of glycemia does not reduce macrovascular events suggesting other mediators may participate to increase the residual cardiovascular risk in diabetic patients ${ }^{7}$. Accumulating evidence indicated that adipose tissue secretes several bioactive mediators termed adipokines, which link metabolic disorders and atherosclerotic cardiovascular diseases ${ }^{8}$. Omentin is among the antiinflammatory and anti-atherogenic adipokine that has potentially beneficial effects on glucose metabolism and cardiovascular disorders ${ }^{9} 10$. However, recent studies indicate a paradoxical association between omentin and cardiovascular events 11121314 . In this study, we investigated the association of circulating omentin levels and endothelial function in the elderly subjects with different glucose metabolism conditions, at the aim of finding potential biomarkers recommended for the clinical use in the prevention or treatment of diabetic patients who at high risk of developing cardiovascular diseases.

\section{Materials And Methods}

\subsection{Population}

We recruited a total of 162 elderly patients with different blood glucose levels at Geriatric Department of Ruijin hospital affiliated to Shanghai Jiao Tong University School of Medicine between December 2017 and May 2019. According to the plasma glucose criteria, patients were divided into three groups: 48 subjects with normal glucose tolerance (NGT), 56 subjects with impaired glucose regulation (IGR, also known as prediabetes), including 28 subjects with impaired glucose tolerance (IGT) and 28 subjects with impaired fasting glucose (IFG), 58 subjects with type 2 diabetes mellitus (T2DM). Diagnoses were made 
according to the American Diabetes Association Criteria ${ }^{15}$. A $75 \mathrm{~g}$ oral glucose tolerance test were performed in all subjects. Subjects with fasting plasma glucose(FPG) value $<5.6 \mathrm{mmol} / \mathrm{l}$ and 2 -h plasma glucose (2-h PG) value during a 75-g oral glucose tolerance test (OGTT) $<7.8 \mathrm{mmol} / \mathrm{l}$ are considered as normal glucose tolerance (NGT); IFG is defined as FPG levels between 100 and $125 \mathrm{mg} / \mathrm{dL}$ (between 5.6 and $6.9 \mathrm{mmol} / \mathrm{L}$ ) and IGT as 2-h post-load plasma glucose between 140 and $199 \mathrm{mg} / \mathrm{dL}$ (between 7.8 and $11.0 \mathrm{mmol} / \mathrm{L}$ ). Patients with IGR are defined by the presence of IFG and/or IGT and/or HbA1C 5.7$6.4 \%$. The fasting plasma glucose (FPG) $\geq 126 \mathrm{mg} / \mathrm{dL}$ ( $7.0 \mathrm{mmol} / \mathrm{L}$ ) or the 2-h plasma glucose (2-h PG) value $\geq 200 \mathrm{mg} / \mathrm{dL}(11.1 \mathrm{mmol} / \mathrm{L})$, or $\mathrm{HbA} 1 \mathrm{C}$ criteria $\geq 6.5 \%$ are diagnosed as type 2 diabetes mellitus (T2DM). Patients with type 1 diabetes or other types of diabetes were not included in this study. The clinical characteristics of the three groups are shown in Table 1.

Exclusion criteria: Those with acute cardiovascular and cerebrovascular diseases, acute infectious diseases, chronic heart failure, liver and kidney dysfunction, valvular diseases, arrhythmias, autoimmune diseases, malignancies and hyperthyroidism were excluded in this study.

This study was approved by the Clinical Research Ethics Committee of Ruijin Hospital Shanghai Jiao Tong University School of Medicine. Informed consent was obtained from each participant after the purpose of this study was explained.

\subsection{Anthropometric and biochemical measurements}

The subjects' age, gender, previous history and medication history were recorded by special personnel. Body weight and height were measured in all participators using standard protocols, BMI was calculated according to the following formula: $\mathrm{BMl}=$ weight/height squared $\left(\mathrm{kg} / \mathrm{m}^{2}\right)$. Blood pressure was measured on the right arm after a 10-minute rest for three times, values used in the analysis are the average of three readings taken at 5-minute intervals. Those who has a history of hypertension or whose systolic blood pressure $(\mathrm{SBP}) \geq 140 \mathrm{mmHg}$ and/or diastolic blood pressure $(\mathrm{DBP}) \geq 90 \mathrm{mmHg}$ were defined as hypertension.

Fasting elbow venous blood samples were collected in the morning after 12-h fasting period for biochemical test. Fasting plasma glucose was evaluated by the automated glucose oxidase method. $\mathrm{HbA1c}$ was measured by high-performance liquid chromatography. Serum total cholesterol (TC), lowdensity lipoprotein cholesterol (LDL-C), and high-density lipoprotein cholesterol (HDL-C) and serum triglyceride (TG) concentrations were measured by using enzymatic assays. Insulin resistance was calculated by the homeostasis model assessment of insulin resistance (HOMA-IR), which was automatically calculated by the following formula: HOMA-IR=fasting insulin $(\mu \mathrm{U} / \mathrm{ml}) \times$ fasting glucose $(\mathrm{mmol} / \mathrm{L}) / 22.5$.

Serum concentrations of omentin, adiponectin, TNF-a, IL-6 were determined by an enzyme-linked immunosorbent assay (ELISA) kit (provided by Shanghai Senxiong Bio-Tech CO. Ltd). All standards are strictly handled according to the instructions of the kit. 


\subsection{The assessment of macrovascular function}

All patients underwent assessment of arterial dilator function, following the International Brachial Artery Reactivity Task Force guidelines ${ }^{5}$. Before testing, all subjects were in a fasting state for at least 6 hours, vasoactive drugs (such as angiotensin converting enzyme inhibitors, Calcium antagonist, beta receptor blockers), alcohol, caffeine, tea, cigarettes and nicotine were withheld for more than 12 hours. Subjects rested in the supine position for at least 10 minutes in a quiet and warm (temperature of $22-24^{\circ} \mathrm{C}$ ) room, A 10-MHz multifrequency linear array probe attached to a high-resolution ultrasound machine (Toshiba Artida) was used to acquire images of the right brachial artery. The lumen diameter of the artery was defined as the distance from the echo front edge of the near wall-lumen interface to the echo front edge of the far wall-lumen interface. In brief, the basal diameter of the brachial artery was obtained in the cubital region before inducing forearm ischemia, recorded as D0 (mm). Subsequently, a forearm cuff, positioned $1 \mathrm{~cm}$ above the antecubital fossa, was inflated to $50 \mathrm{mmHg}$ above systolic blood pressure for $5 \mathrm{~min}$ and then released to elicit forearm reactive hyperemia. The diameter at the same point of the artery was recorded as D1 (the maximum dilatation after deflation). Flow mediated dilation of brachial artery (FMD), described as the maximum percent change of the brachial artery diameter during forearm hyperemia compared to the basal diameter, was calculated by the following formula: FMD (\%) = (D1D0)/D0*100. After 15 minutes, the brachial artery returned to the baseline. Brachial artery diameter was monitored continuously until achievement of maximal dilatation after administration of $0.5 \mathrm{mg}$ of sublingual nitroglycerin, which was measured as D2, and Nitrate-mediated dilation of brachial artery (NMD) was expressed as the percent maximal increase of the artery diameter after nitroglycerin compared to baseline $[\mathrm{NMD}(\%)=(\mathrm{D} 2-\mathrm{D} 0) / \mathrm{D} 0 * 100]$. We use flow mediated dilatation (FMD) to quantify vascular endothelial function, while apply nitrate-mediated dilation (NMD) to assess endotheliumindependent dilator function. The value of FMD $\otimes 10 \%$ indicates impaired endothelial function, and the lower the FMD value is, the worse the endothelial function is. The variability for this ultrasound determination of FMD and NMD showed a coefficient of variation of $1.2 \%-4.2 \%$ and $3.97 \pm 0.24 \%$ respectively, suggesting our method has good reproducibility ${ }^{16}$. Throughout the measurement of each individual, the probe was maintained in a fixed position. All operations were performed by the same experienced sonographer who was blind to the subjects' clinical and biochemical characteristics.

\subsection{Statistical analysis}

Date are expressed as the mean and standard deviations (SDs), or median (interquartile range), or as percentage according to the variables were normal distributing or not, which were examined by the Kolmogorov-Smirnov equality of distributions test. Skewed parameters, such as triglycerides levels, HDL$C$, fasting glucose, fasting insulin, HOMA-IR $\ T N F-a$, adiponectin and omentin levels were logarithmically transformed to normalize their distributions before regression analysis. Differences among the three groups were tested using ANOVA for normally variables while Kruskal-Wallis $\mathrm{H}$ test was used for nonnormally distributed variables. Pearson's correlation coefficient was used for calculation of associations between serum omentin levels and clinical factors, so as the FMD. While multiple stepwise linear regression analysis was used to determine significant confounding factors for circulating omentin levels 
and FMD separately. Clinically and statistically significant variables were added in the regression model. Data were analyzed using SPSS 22.0 software. We regarded P value of less than 0.05 as statistically meaningful.

\section{Results}

\subsection{Anthropometric, clinical and biochemical characteristics}

A total of 162 elderly subjects were recruited in our study. The mean age of participators was $78.64 \pm 8.96$ and $84 \%$ were male. Baseline characteristics of subjects stratified by the status of glucose tolerance test (NGT, IGR and T2DM) are presented separately in Table 1. BMI, blood pressure, TG, TC, LDL-C, HDL-C were similar among the three groups. The levels of FPG, FINS, 2 h PG, HbA1c and HOMA-IR were higher in T2DM group compared with NGT and IGR groups. However, no significant differences in terms of FPG, FINS, 2 h-PG, HOMA-IR and HbA1c were detected between NGT and IGR groups. Besides, Subjects with T2DM were more likely to be older, showing higher levels of TNF-a, IL-6 when compared with NGT groups, but no significant differences were detected within them. The median value of the plasma adiponectin and omentin levels in all subjects was $22.61 \mathrm{ug} / \mathrm{ml}$ (range 3.68-80.79ug/ml) and $16.23 \mathrm{ng} / \mathrm{ml}$ (range $2.30-54.47 \mathrm{ng} / \mathrm{ml}$ ) respectively. Adiponectin shows a downward tendency between the three groups, there are no significant difference either. While circulating omentin concentration was significantly decreased in T2DM group compared with NGT group, but no significant difference was found between IGR and NGT group $(19.07 \pm 12.51 \mathrm{ng} / \mathrm{ml}$ versus $17.16 \pm 8.69 \mathrm{ng} / \mathrm{ml}$ versus $12.98 \pm 9.19 \mathrm{ng} / \mathrm{ml} ; \mathrm{P}=0.004)$. Figure 1 .

Table 1 Clinical characteristic of subjects stratified by the status of glucose tolerance 


\begin{tabular}{|lllll|}
\hline & NGT & IGR & T2DM & P \\
\hline N & 48 & 56 & 58 & \\
\hline Age(year) & $75.54 \pm 9.71$ & $79.7 \pm 7.41^{\mathrm{a}}$ & $80.17 \pm 9.2^{\mathrm{a}}$ & 0.016 \\
\hline BMI(kg/m2) & $25.29 \pm 2.6$ & $24.3 \pm 2.49^{\mathrm{a}}$ & $24.45 \pm 2.39$ & 0.103 \\
\hline SBP(mmHg) & $137.63 \pm 15.68$ & $137.34 \pm 12.33$ & $140.57 \pm 17.29$ & 0.463 \\
\hline DBP(mmHg) & $72.42 \pm 10.57$ & $71.88 \pm 7.79$ & $72.97 \pm 10.42$ & 0.834 \\
\hline HbA1c(\%) & $5.68 \pm 0.3$ & $5.84 \pm 0.27$ & $6.45 \pm 1^{\mathrm{ab}}$ & 0.000 \\
\hline FPG (mmol/L) & $5.55 \pm 0.68$ & $5.9 \pm 0.31$ & $6.75 \pm 1.96$ ab & 0.000 \\
\hline FINS (ulU/L) & $9.66 \pm 4.61$ & $9.12 \pm 1.13$ & $12.6 \pm 10.95^{\mathrm{ab}}$ & 0.02 \\
\hline 2 h-PG(mmol/L) & $7.45 \pm 1.89$ & $9 \pm 1.56$ & $10.47 \pm 3.43^{\mathrm{ab}}$ & 0.000 \\
\hline 2 h-INS(ulU/L) & $84.49 \pm 55.35$ & $70.71 \pm 26.97$ & $75.38 \pm 55.14$ & 0.33 \\
\hline HOMA-IR & $2.42 \pm 1.28$ & $2.39 \pm 0.34$ & $4.49 \pm 6.77^{\mathrm{ab}}$ & 0.01 \\
\hline TG(mmol/L) & $1.2 \pm 0.39$ & $1.3 \pm 0.37$ & $1.49 \pm 1.12$ & 0.116 \\
\hline TC(mmol/L) & $4.26 \pm 1.01$ & $4.27 \pm 1.04$ & $3.99 \pm 1$ & 0.256 \\
\hline LDL-C(mmol/L) & $2.52 \pm 0.79$ & $2.63 \pm 0.88$ & $2.32 \pm 0.89$ & 0.161 \\
\hline HDL-C(mmol/L) & $1.28 \pm 0.27$ & $1.14 \pm 0.36$ & $1.28 \pm 0.51$ & 0.115 \\
\hline sd LDL-C(mmol/L) & $0.71 \pm 0.28$ & $0.5 \pm 0.2$ & $0.69 \pm 0.27$ & 0.000 \\
\hline APN(ug/ml) & $24.09 \pm 15.94$ & $20.41 \pm 11.18$ & $23.51 \pm 19.32$ & 0.434 \\
\hline TNF-a(pg/ml) & $39.76 \pm 8.29$ & $41.17 \pm 11.05$ & $44.62 \pm 15.15^{\mathrm{a}}$ & 0.099 \\
\hline IL-6(pg/ml) & $34.57 \pm 11.04$ & $37.29 \pm 11.17$ & $38.38 \pm 15.71$ & 0.31 \\
\hline Omentin(ng/ml) & $19.07 \pm 12.51$ & $17.16 \pm 8.69$ & $12.98 \pm 9.19^{\mathrm{ab}}$ & 0.004 \\
\hline FMD $(\%)$ & $10.55 \pm 7.64$ & $7.1 \pm 5.56^{\mathrm{a}}$ & $6.68 \pm 4.69 \mathrm{a}$ & 0.002 \\
\hline
\end{tabular}

NGT: normal glucose tolerance; IGR: impaired glucose regulation; IFG: impaired fasting glucose tolerance; IGT: impaired glucose tolerance; T2DM: type 2 diabetes mellitus; BMI: body mass index; SBP: systolic blood pressure; DBP: diastolic blood pressure; FPG: fasting plasma glucose; FINS: fasting serum insulin; 2 h PG: 2 h pose glucose load plasma glucose; 2 h-INS: 2 h serum insulin; HbA1c: glycosylated hemoglobin; HOMA-IR: homeostasis model assessment of insulin resistance; TG: triglycerides; TC: total cholesterol; LDL-C: low-density lipoprotein cholesterol; HDL-C: high-density lipoprotein cholesterol; sd LDL-C: small dense LDL-C; APN: adiponectin; TNF-a: tumor necrosis factor a; IL-6: interleukin-6; FMD: flow mediated dilation; NMD: nitrate-mediated dilation. 


\begin{tabular}{|c|c|c|c|c|}
\hline & NGT & IGR & T2DM & $P$ \\
\hline NMD (\%) & $13.74 \pm 8.15$ & $11.18 \pm 7.56$ & $12.82 \pm 8.55$ & 0.262 \\
\hline \multicolumn{5}{|c|}{$\begin{array}{l}\text { NGT: normal glucose tolerance; IGR: impaired glucose regulation; IFG: impaired fasting glucose } \\
\text { tolerance; IGT: impaired glucose tolerance; T2DM: type } 2 \text { diabetes mellitus; BMI: body mass index; } \\
\text { SBP: systolic blood pressure; DBP: diastolic blood pressure; FPG: fasting plasma glucose; FINS: } \\
\text { fasting serum insulin; } 2 \text { h PG: } 2 \text { h pose glucose load plasma glucose; } 2 \text { h-INS: } 2 \text { h serum insulin; } \\
\text { HbA1c: glycosylated hemoglobin; HOMA-IR: homeostasis model assessment of insulin resistance; } \\
\text { TG: triglycerides; TC: total cholesterol; LDL-C: low-density lipoprotein cholesterol; HDL-C: high-density } \\
\text { lipoprotein cholesterol; sd LDL-C: small dense LDL-C; APN: adiponectin; TNF-a: tumor necrosis factor } \\
\text { a; IL-6: interleukin-6; FMD: flow mediated dilation; NMD: nitrate-mediated dilation. }\end{array}$} \\
\hline
\end{tabular}

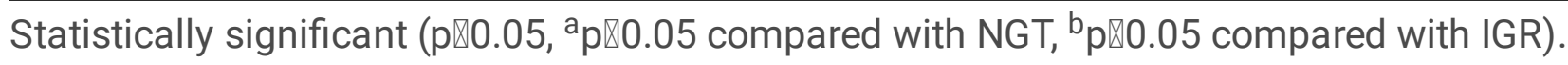

\subsection{Relationships between circulating omentin and clinical data}

The bivariate correlation (Pearson analysis) and linear regression analysis were conducted between circulating omentin levels and clinical parameters and inflammatory factors. Our results indicated that serum omentin concentrations have a negative relationship with $\operatorname{SBP}(r=-0.18, P=0.011), \mathrm{HbA} 1 \mathrm{c} \square \mathrm{r}=-0.222$, $P=0.002 \rrbracket, \log [F P G](r=-0.173, P=0.014), \log [F I N S](r=-0.362, P \bowtie 0.000), \log [T G](r=-0.4 \square P \bigotimes 0.000)$ and

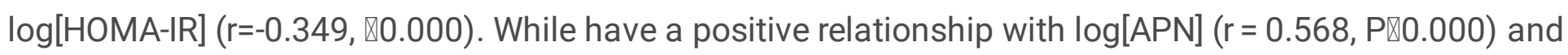
$\log [H D L-C](r=0.288 \square P \otimes 0.000)$. Besides, levels of omentin were negatively associated with TNF- $a$ $(r=-0.146 \square \mathrm{P}=0.032)$. To eliminate the influence of other variables, traditional atherosclerotic risk factors, including age, BMI, SBP, FPG, FINS, HbA1c, TG, HDL-C and levels of TNF-a, IL-6, adiponectin were added to the multiple stepwise regression analysis model, and we finally found that log (FINS), log (HOMA-IR), $\log (\mathrm{TNF}-\mathrm{a})$ and $\log$ (adiponectin) were independently related factors that influenced serum omentin levels. The results were show in Table 2.

When omentin levels were analyzed according to vascular endothelial function status, we found plasma omentin levels were significantly lower in endothelial dysfunction (ED) than normal endothelial function (NEF) in all subjects $(20.12 \pm 13.32 \mathrm{ng} / \mathrm{ml}$ verse $14.44 \pm 8.19 \mathrm{ng} / \mathrm{ml}, \mathrm{P}=0.007)$ Table 3 . Similar result was indicated in participators with NGT, data wasn't showed. Then we conducted an analysis in each subgroup respectively found the plasma omentin levels in IGR subjects with endothelial dysfunction were higher than those of normal endothelial function $(18.511 \pm 9.35 \mathrm{ng} / \mathrm{ml}$ verse $13.46 \pm 5.16 \mathrm{ng} / \mathrm{ml}, \mathrm{P}=$ 0.014). Notably, in T2DM group with endothelial dysfunction, the serum omentin levels were lowest (Fig. 2).

Table 2 Bivariate correlation analysis and multiple regression analysis of plasma omentin levels and study variables 


\begin{tabular}{|c|c|c|c|c|}
\hline & \multicolumn{2}{|c|}{ Correlation coefficient (r) } & \multicolumn{2}{|c|}{ Regression coefficient ( $\beta$ ) } \\
\hline & $r$ & $P$ value & $\beta$ & $P$ value \\
\hline Age(year) & 0.004 & 0.48 & 0.03 & 0.652 \\
\hline $\mathrm{BMI}(\mathrm{kg} / \mathrm{m} 2)$ & 0.035 & 0.329 & 0.106 & 0.096 \\
\hline $\mathrm{SBP}(\mathrm{mmHg})$ & -0.18 & 0.011 & 0.017 & 0.802 \\
\hline $\log (\mathrm{FPG})$ & -0.173 & 0.014 & 0.124 & 0.252 \\
\hline $\log (\mathrm{FINs})$ & -0.362 & 0.000 & -0.207 & 0.006 \\
\hline $2 \mathrm{~h}-\mathrm{PG}(\mathrm{mmol} / \mathrm{L})$ & -0.155 & 0.025 & -0.118 & 0.171 \\
\hline $\mathrm{HbA} 1 \mathrm{c}(\%)$ & -0.222 & 0.002 & -0.101 & 0.257 \\
\hline $\log (\mathrm{HOMA}-\mathrm{IR})$ & -0.349 & 0.000 & & \\
\hline log(triglycerides) & -0.4 & 0.000 & -0.201 & 0.004 \\
\hline $\log (\mathrm{HDL}-\mathrm{C})$ & 0.288 & 0.000 & 0.051 & 0.475 \\
\hline log(adiponectin) & 0.568 & 0.000 & 0.425 & 0.000 \\
\hline $\log (T N F-a)$ & -0.146 & 0.032 & -0.148 & 0.026 \\
\hline \multirow[t]{2}{*}{ IL-6(pg/ml) } & -0.031 & 0.35 & -0.034 & 0.603 \\
\hline & & & $\mathrm{R} 2(\mathrm{P})$ & $0.454(0.008)$ \\
\hline
\end{tabular}

Table 3 Comparison of serum omentin levels in each subgroup according to vascular endothelial function status.

\begin{tabular}{|lllllll|}
\hline \multicolumn{3}{|c}{ All subjects } & \multicolumn{5}{c}{ IGT } & & T2DM & \\
\hline & NEF & ED & NEF & ED & NEF & ED \\
\hline N & 51 & 111 & 15 & 41 & 13 & 45 \\
\hline $\begin{array}{l}\text { Omentin } \\
\text { (ng/ml) }\end{array}$ & $20.12 \pm$ & $14.44 \pm$ & $13.46 \pm$ & $18.511 \pm$ & $19.30 \pm$ & $11.16 \pm$ \\
\hline P & 0.007 & 8.19 & 5.16 & 9.35 & 14.21 & 6.24 \\
\hline NEF: normal endothelial function, ED: endothelial dysfunction. & & & \\
\hline
\end{tabular}




\subsection{Association of circulating omentin and endothelial dysfunction}

Patients with abnormal glucose metabolism show different degrees of vascular endothelial dysfunction, Lower flow-mediated dilation (FMD) of the brachial artery was found in IGR and T2DM groups compared with NGT group, subjects with T2DM shows the lowest levels of FMD (10.55 \pm 7.64 verse $7.1 \pm 5.56$ verse $6.68 \pm 4.69, P=0.002)$ Table 1 . While no statistically significant difference was observed in terms of NMD between the three groups $(P=0.262)$. To explore the determined factors of vascular endothelial dysfunction, univariate and multivariate analysis were performed.

First, we explored metabolic factors and adipokines (including anti-inflammatory factors omentin and adiponectin and inflammatory factors TNF-a, IL-6) associated with FMD in all subjects. FMD significantly and negatively associated with SBP, HbA1c, log (FPG), log (HOMA-IR), log (INS) and IL-6, while have a positive relationship with log (omentin) which was indicated by Pearson analysis. Multivariate analyses showed that higher SBP, FINS and IL-6 levels were independent determinants of lower FMD. No significant association was found between $\log (o m e n t i n)$ and FMD after adjusting former mentioned variables in all subjects (Table 4).

To further study the relationship between plasma omentin levels and FMD, multiple regression analyses were performed separately in the subgroups. Even after adjusting for age, BMI, SBP, log (FPG), log (INS), $\log ($ HOMA-IR), $\log (T G), \log (H D L-C), \log (A P N), \log$ (TNF-a), and IL-6, log (omentin) was independent related factor that influenced FMD $(\beta=-0.346, P=0.01)$ in IGR subjects, but not in the subgroups NGT and T2DM. In T2DM group, The effect of glycosylated hemoglobin on vascular dysfunction is greater than that of lower omentin levels $(\beta=-0.525, P=0.009)$. Data was show in Table 5.

Table 4 Bivariate correlation analysis and multiple regression analysis of FMD and study variables in all subjects. 


\begin{tabular}{|lllll|}
\hline & \multicolumn{2}{l}{ Correlation coefficient $(r)$} & \multicolumn{2}{l|}{ Regression coefficient ( $\beta)$} \\
\hline & $r$ & P value & $\beta$ & P value \\
\hline Age (year) & -0.041 & 0.302 & 0.003 & 0.97 \\
\hline $\mathrm{BMI}(\mathrm{kg} / \mathrm{m} 2)$ & -0.049 & 0.267 & -0.037 & 0.621 \\
\hline $\mathrm{SBP}(\mathrm{mmHg})$ & -0.291 & 0.000 & -0.221 & 0.005 \\
\hline $\mathrm{HbA1c}(\%)$ & -0.242 & 0.001 & -0.104 & 0.325 \\
\hline $\log (\mathrm{FPG})$ & -0.225 & 0.002 & 0.02 & 0.859 \\
\hline $\log (\mathrm{FINS})$ & -0.34 & 0.000 & -0.247 & 0.006 \\
\hline $\log (\mathrm{TG})$ & -0.088 & 0.132 & -0.052 & 0.539 \\
\hline $\log (\mathrm{HDL}-\mathrm{C})$ & 0.124 & 0.058 & 0.099 & 0.235 \\
\hline $\log (\mathrm{APN})$ & 0.116 & 0.071 & -0.036 & 0.724 \\
\hline $\log (\mathrm{TNF}-\mathrm{a})$ & -0.062 & 0.215 & 0.06 & 0.452 \\
\hline $\log (\mathrm{omentin})$ & 0.151 & 0.028 & -0.026 & 0.787 \\
\hline $\mathrm{IL}-6(\mathrm{pg} / \mathrm{ml})$ & -0.237 & 0.001 & -0.283 & 0.000 \\
\hline & & & $\beta(\mathrm{P})$ & $0.243(0.000)$ \\
\hline
\end{tabular}

Table 5 Multiple regression analysis for the determinants of FMD in NGT, IGT and T2DM subjects. 


\begin{tabular}{|lllllll|}
\hline & NGT & & IGR & & T2DM \\
\hline & $\beta$ & P value & $\beta$ & P value & $\beta$ & P value \\
\hline Age (year) & -0.15 & 0.24 & -0.109 & 0.392 & 0.064 & 0.684 \\
\hline BMI(kg/m2) & -0.329 & 0.029 & 0.161 & 0.232 & 0.032 & 0.818 \\
\hline SBP (mmHg) & -0.237 & 0.107 & -0.356 & 0.007 & 0.068 & 0.66 \\
\hline HbA1c(\%) & 0.224 & 0.12 & -0.226 & 0.073 & -0.525 & 0.009 \\
\hline Log (FPG) & 0.112 & 0.463 & -0.348 & 0.025 & 0.317 & 0.112 \\
\hline $\log ($ FINs) & -0.419 & 0.016 & -0.214 & 0.097 & -0.073 & 0.658 \\
\hline $\log$ (triglycerides) & -0.023 & 0.865 & 0.043 & 0.709 & 0.025 & 0.894 \\
\hline Log (HDL-C) & 0.036 & 0.789 & 0.063 & 0.619 & 0.393 & 0.019 \\
\hline $\log ($ adiponectin) & 0.01 & 0.966 & -0.216 & 0.155 & -0.347 & 0.253 \\
\hline $\log ($ TNF-a) & -0.212 & 0.169 & -0.252 & 0.097 & 0.147 & 0.41 \\
\hline $\log ($ omentin) & 0.025 & 0.922 & -0.346 & 0.01 & 0.365 & 0.183 \\
\hline IL-6(pg/ml) & -0.282 & 0.043 & -0.076 & 0.577 & -0.249 & 0.099 \\
\hline R2(P) & $0.594(0.000)$ & $0.549(0.000)$ & $0.353(0.042)$ \\
\hline
\end{tabular}

\section{Discussion}

Three major findings are emerged from this study: (1) Individuals diagnosed with T2DM and IGR exhibited lower omentin levels, while levels of inflammatory factors like TNF- $a$ and IL-6 levels were on the rise, which demonstrated that diabetes is not just a state of hyperglycemia but a systemic disorder with chronic inflammation and adipose tissue dysfunction. (2) Diabetic patients show depressed vascular function, mainly refers to endothelial dysfunction, even in the prediabetes status (IGR). (3) Plasma omentin levels were positively associated with FMD, while in subjects with IGR, omentin showed a significantly and negatively relationship with FMD after adjustment for traditional cardiovascular risk factors. Which may suggest higher omentin concentrations in individuals at risk of atherosclerosis reflecting a compensatory mechanism in early stage of diabetes. Our findings may lay a foundation for studying the pathogenesis of early vascular endothelial damage in prediabetic individuals and new therapies to retard the progression of diabetic cardiovascular complications.

Diabetes is a prominent global public health problem with its continuing growth in rates of incidence and prevalence, it is quite common in the elderly. People with diabetes have an increased risk of developing numbers of serious life-threatening health problems and lowering the quality of life. Cardiovascular 
diseases and ischemic stroke are the most common ones. Previous studies showed that hyperglycemia, insulin resistance, oxidative stress and MicroRNA all participate in the development of micro- and macrovascular complications in diabetes ${ }^{17}$. Besides, adiposity seems to modify the proportional effect of diabetes on risk of vascular diseases via the production of various adipokines.

Omentin is a novel adipokine identified from a visceral omental adipose tissue cDNA library in 2006, which was composed of 313 amino acids, and mainly expressed in visceral adipose tissue. More specifically, it is expressed in visceral adipose stromal vascular cells, rather than in subcutaneous adipose tissue ${ }^{9}$. Accumulating evidence indicates that the obese state is characterized by chronic lowgrade inflammation and adipose tissue dysfunction, which leads to the initiation and progression of obesity-related disorders, such as T2DM, hypertension, and atherosclerosis ${ }^{18}$. We found in the present study, circulating omentin levels were lower in IGR group compared with NGT and were lowest in individuals with T2DM group. This was in line with a recently meta-analysis contains of twenty-eight studies with 3354 participants in which demonstrated serum omentin level is significantly lower in T2DM and IGR patients ${ }^{19}$. Decreased serum omentin level could cause a reduction in insulin stimulated glucose uptake in insulin-sensitive tissues in T2DM patients ${ }^{20}$. This may partly explain the state of insulin resistance presented in T2DM. It has also been reported that glucose and insulin could decrease mRNA expression of omentin and omentin protein production in adipose tissue ${ }^{20}$.Additionally, our data showed that omentin concentration were inversely correlated with multiple cardiovascular risk factors including SBP, glucose levels, insulin resistance and disadvantaged lipid levels. while had a positive relationship with HDL-C. Which were in accordance with other studies ${ }^{21}$. Previous study shows omentin is an antiinflammatory molecule 222312 . Hideyuki et al. further indicated that omentin exerts anti-inflammatory effects in vascular endothelial cells by preventing TNF-a induced cyclooxygenase-2 (COX-2) expression via inhibiting JNK signal presumably through activation of AMPK/eNOS/NO pathways ${ }^{22}$. In our study, we found TNF-a negatively associated with circulating omentin concentrations, even after adjusting the traditional atherosclerotic risk factors, including age, BMI, SBP, FPG, FINS, HbA1c, TG and HDL-C. Which shows the lower circulating omentin value may in part contributed to the chronic low-glade inflammatory status in subjects with diabetes.

Endothelial dysfunction is implicated in a variety of cardiovascular diseases characterized by a reduced production and availability of nitric oxide (NO). The measurement of flow-mediated dilation (FMD) of the brachial artery after forearm ischemia is supposed to be a non-invasive method to assess endothelial production and release of NO, which has been widely used to assess vascular function. At present, it is generally accepted that the value of FMD less than $10 \%$ indicates impaired endothelial function, and the lower the FMD value is, the worse the endothelial function is ${ }^{5}$. Besides, FMD has demonstrated to be a good predictor of future cardiovascular events. A current meta-analysis found that a 1 SD increase or decrease of FMD was associated with $50 \%$ lower risk or doubled risk of cardiovascular events 2425 . In our study, FMD was decreased in subjects with T2DM and IGR compared with NGT group, no difference was found in terms of NMD, which shows that the patients with abnormal glucose metabolism had different degrees of vascular endothelial dysfunction rather than vascular smooth muscle dysfunction. 
Hypertension, hyperglycemia, and insulin resistance are traditional risk factors known to have a key role in the pathogenic mechanisms of cardiovascular diseases. Inflammatory cytokines, such as TNF-a and

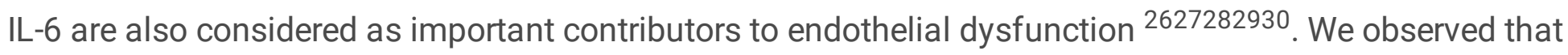
FMD significantly and negatively associated with SBP, HbA1c, log (FPG), log (HOMA-IR) and log (INS). A relationship has also been suggested to exist between inflammation and endothelial dysfunction in our study. Even after adjustment for other factors, higher IL-6 levels were independent determinants of lower FMD, this was in accordance with Lee. et. al, who indicated that TNF-a and IL-6 exacerbate oxidative stress and reduce phosphorylation of endothelial nitric oxide synthase (eNOS), thereby contributing to coronary endothelial dysfunction in T2DM mice. Omentin possesses anti-atherosclerotic and vasculoprotective properties. Maruyama S, et al indicated that omentin promotes endothelial cell function and revascularization in response to ischemia through its ability to stimulate an Akt-eNOS signaling pathway ${ }^{31}$. Vitro studies indicated omentin might play a preventive role in the pathogenesis of obesity and diabetes-related vascular complications ${ }^{12}{ }^{32}$. In agreement with previous cross-sectional studies reported that circulating omentin concentrations were lower in people with coronary artery disease or ischemic stroke compared with individuals without cardiovascular disease ${ }^{33} 34$, our study demonstrated that plasma omentin levels decreased in subjects with the early stage of atherosclerosis, i.e. endothelial dysfunction, especially in patients with diabetes. After adjustment for other factors, no significant association was found between log(omentin) and FMD in all subjects and T2DM group. However, in IGR group, the higher circulating omentin levels are independent determining factors for endothelial dysfunction. Although the detailed mechanisms are not yet completely understood. Omentin could be upregulated in response to metabolic and inflammatory stimuli that contribute to vascular endothelial dysfunction in the early stage of diabetes. One potential explanation for the adverse association between omentin and endothelial dysfunction in IGR subjects is higher omentin levels in individual at risk of atherosclerosis reflecting a compensatory mechanism, just like there exist insulin resistance in prediabetes, but this self-regulating mechanism is insufficient to protect against the onset of endothelial dysfunction when hyperglycemia persists or reaches the diagnostic criteria for diabetes, then lower omentin levels in diabetic patients appears. If this were true, associations could be explained by reverse causation because higher omentin levels would represent early symptoms of prediabetic endovascular injury.

\section{Limitations}

Our study both has strengths and limitations. It is the first study to demonstrates that circulating omentin levels strongly predicts endothelial function in elderly patients with impaired glucose regulation independently from traditional cardiovascular risk factors and from adiponectin level. Additionally, our study mainly focused on patients with impaired glucose regulation (IFG and IGT), which was considered as pre-diabetes. The main limitation of our study was its cross-sectional design, we are not yet sure of the causal relationship between the plasma omentin concentration and diabetic vascular dysfunction. In addition, our sample size was too small to make the case, besides, the elderly mainly centered in Shanghai may have regional differences and are not representative. Finally, we did not take the duration 
of diabetes and medication histories into account when we included the study subjects, which may affect the experimental results.

\section{Conclusion}

A decreased omentin levels had been suggested to be a biomarker of glucose metabolic disorders and potentially cardiovascular risk. Higher circulating omentin level are independent determining factors for endothelial dysfunction in IGR subjects and may be a compensatory mechanism for early diabetic vascular injury. which suggest omentin may act as potential biomarkers for assessing endothelial dysfunction and identifying high risk of cardiovascular complications among asymptomatic prediabetic individuals.

\section{Abbreviations}

NGT: normal glucose tolerance; IGR: impaired glucose regulation; IFG: impaired fasting glucose tolerance; IGT: impaired glucose tolerance; T2DM: type 2 diabetes mellitus; BMI: body mass index; SBP: systolic blood pressure; DBP: diastolic blood pressure; FPG: fasting plasma glucose; FINS: fasting serum insulin; 2h PG: $2 \mathrm{~h}$ pose glucose load plasma glucose; $2 \mathrm{~h}$-INS: $2 \mathrm{~h}$ serum insulin; HbA1c: glycosylated hemoglobin; HOMA-IR: homeostasis model assessment of insulin resistance; TG: triglycerides; TC: total cholesterol; LDL-C: low-density lipoprotein cholesterol; HDL-C: high-density lipoprotein cholesterol; sd LDL-C: small dense LDL-C; APN: adiponectin; TNF-a: tumor necrosis factor a; IL-6: interleukin-6; FMD: flow mediated dilation; NMD: nitrate-mediated dilation; NEF: normal endothelial function; ED: endothelial dysfunction; NO: nitric oxide; eNOS: endothelial nitric oxide synthase; COX-2: cyclooxygenase『AMPK: 5'-AMPactivated protein kinase.

\section{Declarations}

\section{Ethics approval and consent to participate}

This study was approved by the Clinical Research Ethics Committee of Ruijin Hospital Shanghai Jiao Tong University School of Medicine. Ethics committee reference number : Grand No 113 (2017). Informed consent was obtained from each participant after the purpose of this study was explained.

\section{Conflict of interest}

The authors declare that they have no conflict of interest to disclose.

\section{Consent for publication}

Not applicable

\section{Availability of data}


All data generated or analyzed during this study are included in this article.

\section{Founding}

This work was supported by Shanghai Shen Kang Medical Development Center Promotes Municipal Hospitals' Clinical Skills and Innovation Ability $₫$ Grant No. 16CR4013A

\section{Author contributions:}

Dongdong Ye and Wei Liang conceived of the study and drafted the manuscript. Wenqi Pan participated in its design and performed the statistical analysis. Zhengjia Su selected and enrolled the patients and performed the exams. All authors have read and approved the final manuscript.

\section{Author details}

Dongdong Ye: Shanghai Jiao Tong University School of Medicine, 227 South Chongqing Road, Shanghai, 200025, China. Wei Liang and Zhengjia Su: Department of geriatrics, Rui Jin Hospital, Shanghai Jiao Tong University School of Medicine, 197 Rui Jin Er Road, Shanghai, 200025, China. Wenqi Pan: Department of cardiology, Rui Jin Hospital, Shanghai Jiao Tong University School of Medicine, 197 Rui Jin Er Road, Shanghai, 200025, China.

\section{Acknowledgements}

The authors thank Dr, Yun Hu for carrying on the endothelial function test. The authors also thank Haitao Zhang.

\section{References}

1. Sarwar N, Gao P, Kondapally Seshasai SR, et al. Diabetes mellitus, fasting blood glucose concentration, and risk of vascular disease: A collaborative meta-analysis of 102 prospective studies. Lancet. 2010;375(9733):2215-2222. doi:10.1016/S0140-6736(10)60484-9

2. Palmer SC, Mavridis D, Nicolucci A, et al. Comparison of clinical outcomes and adverse events associated with glucose-lowering drugs in patients with type 2 diabetes a meta-analysis. JAMA - J Am Med Assoc. 2016;316(3):313-324. doi:10.1001/jama.2016.9400

3. International Diabetes Federation, IDF Diabetes Atlas 8th edition. Eighth Edition 2017; 2017. doi:http://dx.doi. org/10.1016/S0140-6736(16)31679-8.

4. Schächinger V, Britten MB, Zeiher AM. Prognostic impact of coronary vasodilator dysfunction on adverse long- term outcome of coronary heart disease. Circulation. 2000;101(16):1899-1906. doi:10.1161/01.CIR.101.16.1899

5. Corretti MC, Anderson TJ, Benjamin EJ, et al. Guidelines for the ultrasound assessment of endothelial-dependent flow-mediated vasodilation of the brachial artery: A report of the international 
brachial artery reactivity task force. J Am Coll Cardiol. 2002;39(2):257-265. doi:10.1016/S07351097(01)01746-6

6. Morris SJ, Shore AC. Skin blood flow responses to the iontophoresis of acetylcholine and sodium nitroprusside in man: Possible mechanisms. J Physiol. 1996;496(2):531-542. doi:10.1113/jphysiol.1996.sp021704

7. Sattar N, Wannamethee SG, Forouhi NG. Novel biochemical risk factors for type 2 diabetes: Pathogenic insights or prediction possibilities? Diabetologia. 2008;51(6):926-940. doi:10.1007/s00125-008-0954-7

8. Ouchi N. Adipocytokines in cardiovascular and metabolic diseases. J Atheroscler Thromb. 2016;23(6):645-654. doi:10.5551/jat.34918

9. Yang RZ, Lee MJ, Hu H, et al. Identification of omentin as a novel depot-specific adipokine in human adipose tissue: Possible role in modulating insulin action. Am J Physiol - Endocrinol Metab. 2006;290(6):1253-1261. doi:10.1152/ajpendo.00572.2004

10. Kataoka Y, Shibata R, Ohashi K, et al. Omentin prevents myocardial ischemic injury through AMPactivated protein kinase- and akt-dependent mechanisms. J Am Coll Cardiol. 2014;63(24):2722-2733. doi:10.1016/j.jacc.2014.03.032

11. Niersmann C, Carstensen-Kirberg M, Maalmi H, et al. Higher circulating omentin is associated with increased risk of primary cardiovascular events in individuals with diabetes. Diabetologia. 2019. doi:10.1007/s00125-019-05017-2

12. laZhong X, Li X, Liu F, Tan H, Shang D. Omentin inhibits TNF-a-induced expression of adhesion molecules in endothelial cells via ERK/NF-KB pathway. Biochem Biophys Res Commun. 2012;425(2):401-406. doi:10.1016/j.bbrc.2012.07.110

13. Nishimura $M$, Morioka $T$, Hayashi $M$, et al. Plasma omentin levels are inversely associated with atherosclerosis in type 2 diabetes patients with increased plasma adiponectin levels: A crosssectional study. Cardiovasc Diabetol. 2019;18(1):1-10. doi:10.1186/s12933-019-0973-3

14. Saely $\mathrm{CH}$, Leiherer A, Muendlein A, et al. High plasma omentin predicts cardiovascular events independently from the presence and extent of angiographically determined atherosclerosis. Atherosclerosis. 2016;244:38-43. doi:10.1016/j.atherosclerosis.2015.10.100

15. Care D, Suppl SS. Classification and diagnosis of diabetes: Standards of medical care in Diabetesd2018. Diabetes Care. 2018;41(January):S13-S27. doi:10.2337/dc18-S002

16. Meyer MF, Lieps D, Schatz H, Pfohl M. Impaired flow-mediated vasodilation in type 2 diabetes: Lack of relation to microvascular dysfunction. Microvasc Res. 2008;76(1):61-65. doi:10.1016/j.mvr.2008.03.001

17. Paneni F, Beckman JA, Creager MA, Cosentino F. Diabetes and vascular disease: Pathophysiology, clinical consequences, and medical therapy: Part i. Eur Heart J. 2013;34(31):2436-2446. doi:10.1093/eurheartj/eht149

18. Sengul E, Duygulu G, Dindar S, Bunul F. Serum omentin-1, inflammation and carotid atherosclerosis in patients with non-diabetic chronic kidney disease. Ren Fail. 2013;35(8):1089-1093. 
doi:10.3109/0886022X.2013.817256

19. Schakel W, Bode C, Elsman EBM, et al. The association between visual impairment and fatigue: a systematic review and meta-analysis of observational studies. Ophthalmic Physiol Opt. 2019;39(6):399-413. doi:10.1111/opo.12647

20. Pan HY, Guo L, Li Q. Changes of serum omentin-1 levels in normal subjects and in patients with impaired glucose regulation and with newly diagnosed and untreated type 2 diabetes. Diabetes Res Clin Pract. 2010;88(1):29-33. doi:10.1016/j.diabres.2010.01.013

21. Batista CMDS, Yang R, Lee M, et al. Omentin Plasma Levels and Gene Expression Are Decreased in Obesity. 2007;56(June):1655-1661. doi:10.2337/db06-1506.AFDS

22. Yamawaki H, Kuramoto J, Kameshima S, Usui T, Okada M, Hara Y. Omentin, a novel adipocytokine inhibits TNF-induced vascular inflammation in human endothelial cells. Biochem Biophys Res Commun. 2011;408(2):339-343. doi:10.1016/j.bbrc.2011.04.039

23. Tan BK, Adya R, Randeva HS. Omentin: A Novel Link Between Inflammation, Diabesity, and Cardiovascular Disease. Trends Cardiovasc Med. 2010;20(5):143-148. doi:10.1016/j.tcm.2010.12.002

24. Matsuzawa Y, Kwon TG, Lennon RJ, Lerman LO, Lerman A. Prognostic value of flow-mediated vasodilation in brachial artery and fingertip artery for cardiovascular events: A systematic review and meta-analysis. J Am Heart Assoc. 2015;4(11):1-15. doi:10.1161/JAHA.115.002270

25. Green DJ, Jones $H$, Thijssen D, Cable NT, Atkinson G. Flow-mediated dilation and cardiovascular event prediction: Does nitric oxide matter? Hypertension. 2011;57(3):363-369. doi:10.1161/HYPERTENSIONAHA.110.167015

26. Lee J, Lee S, Zhang H, Hill MA, Zhang C, Park Y. Interaction of IL-6 and TNF-a contributes to endothelial dysfunction in type 2 diabetic mouse hearts. PLoS One. 2017;12(11):1-17. doi:10.1371/journal.pone.0187189

27. Gao X, Xu X, Belmadani S, et al. TNF-a contributes to endothelial dysfunction by upregulating arginase in ischemia/reperfusion injury. Arterioscler Thromb Vasc Biol. 2007;27(6):1269-1275. doi:10.1161/ATVBAHA.107.142521

28. Huang Y, Yan L, Rong S, Haller H, Kirch T. TNF-a induces endothelial dysfunction via PKC- dependent NADPH oxidase activation. J Huazhong Univ Sci Technol - Med Sci. 2012;32(5):642-647. doi:10.1007/s11596-012-1011-9

29. Aljada A, Ghanim H, Assian E, Dandona P. Tumor necrosis factor-a inhibits insulin-induced increase in endothelial nitric oxide synthase and reduces insulin receptor content and phosphorylation in human aortic endothelial cells. Metabolism. 2002;51(4):487-491. doi:10.1053/meta.2002.31339

30. Nawawi H, Osman NS, Annuar R, Khalid BAK, Yusoff K. Soluble intercellular adhesion molecule-1 and interleukin-6 levels reflect endothelial dysfunction in patients with primary hypercholesterolaemia treated with atorvastatin. Atherosclerosis. 2003;169(2):283-291. doi:10.1016/S0021-9150(03)00193$\mathrm{X}$ 
31. Maruyama S, Shibata R, Kikuchi R, et al. Fat-derived factor omentin stimulates endothelial cell function and ischemia-induced revascularization via endothelial nitric oxide synthase-dependent mechanism. J Biol Chem. 2012;287(1):408-417. doi:10.1074/jbc.M111.261818

32. Hiramatsu-ito $M$, Shibata $R$, Ohashi $K$, et al. Omentin attenuates atherosclerotic lesion formation in apolipoprotein E-deficient mice. 2016:107-117. doi:10.1093/cvr/cvv282

33. Zhao L. Serum omentin-1 levels are inversely associated with the presence and severity of coronary artery disease in patients with metabolic syndrome. 2011;16(September):657-662. doi:10.3109/1354750X.2011.622789

34. Yue J, Chen J, Wu Q, et al. Serum levels of omentin-1 association with early diagnosis, lesion volume and severity of acute ischemic stroke. Cytokine. 2018;111(May):518-522.

doi:10.1016/j.cyto.2018.05.026

\section{Figures}

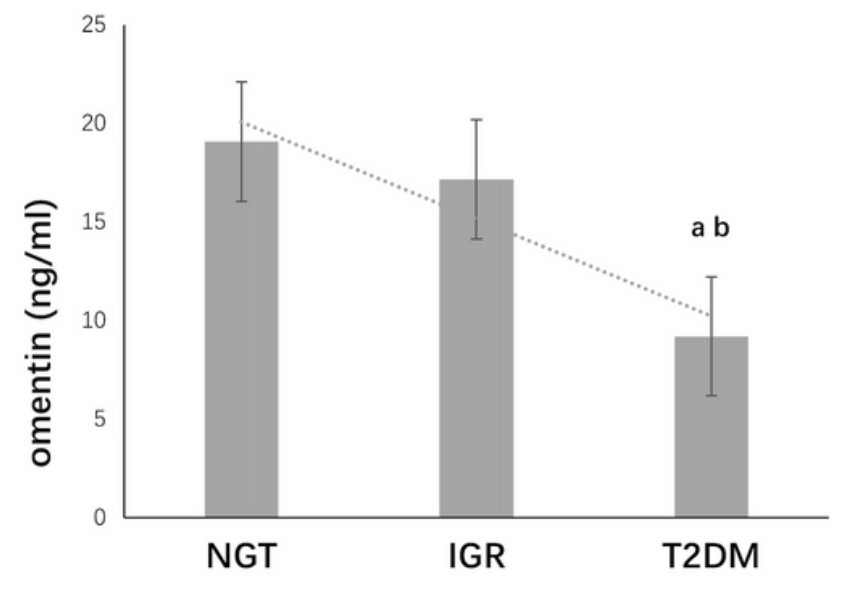

(A)

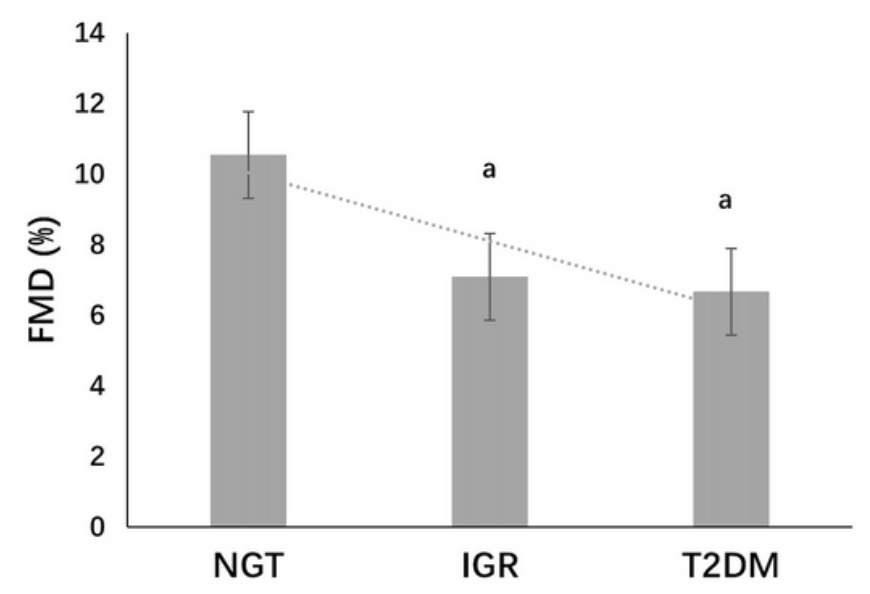

(B)

\section{Figure 1}

Comparison of plasma omentin levels (A) or FMD (B) between NGT, IGR and T2DM. Statistically

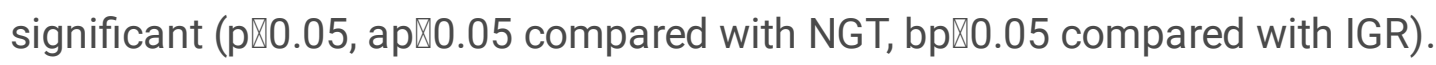




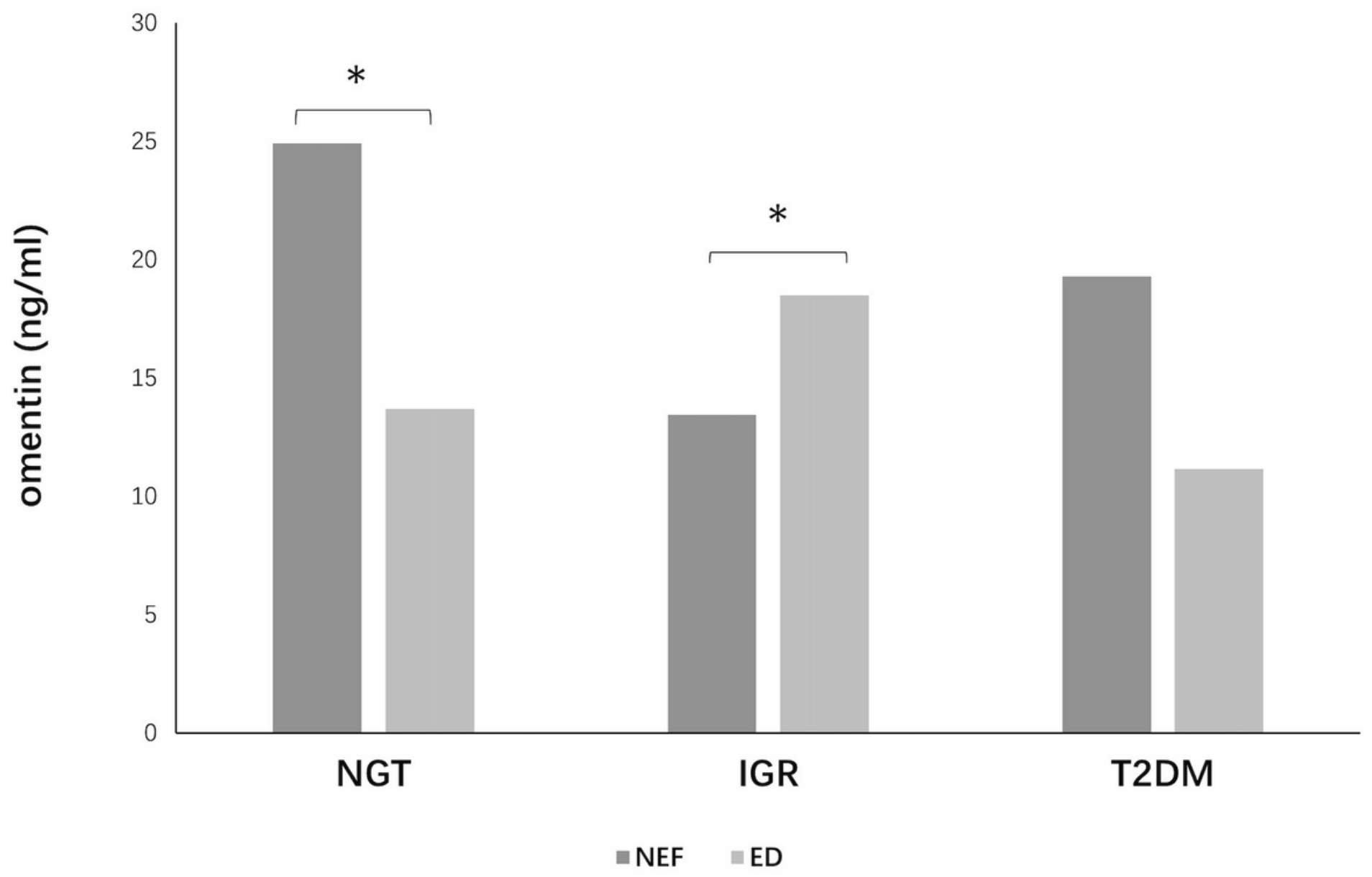

Figure 2

Comparison of serum omentin levels in each subgroup according to vascular endothelial function status. NEF: normal endothelial function, ED: endothelial dysfunction. ${ }^{*},<0.05$ vs endothelial dysfunction group by T-test. 